\title{
An LMI characterization of polynomial parameter-dependent Lyapunov functions for robust stability
}

\author{
R. C. L. F. Oliveira, V. J. S. Leite, M. C. de Oliveira and P. L. D. Peres
}

\begin{abstract}
This paper investigates the robust stability of continuous-time, time-invariant linear uncertain systems in polytopic domains. Robust stability is checked by constructing a quadratic parameter-dependent Lyapunov function. The matrix associated with this quadratic Lyapunov function is a polynomial function of the uncertain parameters, expressed as a particular polynomial matrix involving powers of the dynamic matrix of the system and one symmetric matrix to be determined. The degree of this polynomial matrix function is arbitrary. Finsler's Lemma is used to lift the obtained stability conditions into a larger space in which sufficient stability tests can be developed in the form of Linear Matrix Inequalities (LMIs), which must be verified at the vertices of the uncertainty polytopic domain. Examples illustrate the method, which is compared with similar results in the literature by means of random numerical experiments.
\end{abstract}

\section{INTRODUCTION}

Robust stability of linear uncertain systems through the use of Lyapunov functions has been the subject of several studies in the systems and control literature. The concept of quadratic stability [1], where a single constant Lyapunov matrix is used to verify robust stability throughout the entire uncertainty domain, has been widely applied to robust stability analysis, and robust control and filtering design. This may be mainly due to the simplicity of the resulting conditions, which in most cases can be cast as Linear Matrix Inequalities (LMIs) [2]. Problems with LMI constraints can be formulated as semi-definite programs, which can be solved in polynomial-time using interior-point algorithms [3], [4]. Unfortunately, simplicity has its price, and the results obtained with the help of the quadratic stability concept are typically conservative.

In the last decade, new classes of Lyapunov functions have been proposed and studied in an attempt to reduce conservatism in robust stability analysis. The so-called parameterdependent Lyapunov functions pursue this goal by allowing certain parameters on the Lyapunov functions to depend explicitly on the uncertain parameters.

Concerning continuous time-invariant uncertain systems, sufficient LMI conditions assuring the existence of a

This work is partially supported by the Brazilian agencies CAPES, CNPq and FAPESP.

R. C. L. F. Oliveira and P. L. D. Peres are with the School of Electrical and Computer Engineering, University of Campinas, CP 6101, 13081-970, Campinas, SP, Brazil \{ricfow, peres\}adt.fee.unicamp.br.

V. J. S. Leite is with the UnED Divinópolis - CEFET-MG, R. Monte Santo, 319 - 35502-036, Divinópolis, MG, Brazil valter@iv.cefetmg.br.

M. C. de Oliveira is with the University of California San Diego, Department of Mechanical and Aerospace Engineering, La Jolla, CA, 92093-0411, USA mauricio@ucsd. edu. quadratic Lyapunov function with an affine parameter dependent Lyapunov matrix appeared in [5], with the introduction of some extra matrix variables, in [6], using algebraic properties and an extra set of LMIs, in [7], which extended the results of [5] to a generic region inside the complex plane, and in [8], using extra matrices and LMIs. A related technique is proposed in [9], where an alternative procedure is used to introduce extra matrix variables.

More recently, parameter dependent Lyapunov matrices which are polynomials of higher degree have been studied, for instance, in [10]. In this work, a sequence of LMI conditions which approaches necessity asymptotically is investigated. However, the required computational burden becomes rapidly prohibitive. In [11], robust stability conditions were expressed through a complete square matrix representation of homogeneous matrix forms, providing increasingly accurate characterizations of robust stability. We have recently become aware of the work [12], which uses methods that are very simmilar to the ones proposed in this paper.

It is also worth mentioning the recent works based on the optimization of positive polynomials over compact sets [13], [14], such as [15], [16], [17], which results in problems that can be solved as a sequence of LMI relaxations. These relaxations work by introducing more free variables and larger inequalities in the LMI conditions, which improves the resolution of such tests. The challenge is to adequately trade-off the computational effort with the reduction in conservativeness of the robust stability analysis.

In this paper, we investigate robust stability of continuoustime, time-invariant linear uncertain systems. Uncertainty is represented as a polytope in the state space. We test stability using a quadratic polynomial parameter-dependent Lyapunov function, where the matrix describing the quadratic function depends polynomially on the uncertain parameter. Differently from previous works, this matrix is not a generic polynomial, but a rather particular polynomial of arbitrary degree involving the dynamic matrix of the uncertain system and a symmetric matrix to be determined. The reason for adopting such particular form of the polynomial Lyapunov matrix is our ability to use Finsler's Lemma to lift the obtained stability conditions into equivalent yet much simpler inequalities in a larger dimensional space. From these lifted conditions, robust stability tests are developed as LMIs that need to be satisfied at the vertices of the uncertainty polytopic domain. Our results provides the user with a family of LMI conditions of increasing precision and complexity, which grows with the desired degree of the polynomial parameterdependent Lyapunov function. 


\section{Polynomial Parameter-Dependent Lyapunov FUNCTIONS}

Consider the linear time-invariant continuous-time uncertain system described in state space by the differential equation

$$
\dot{x}=A(\alpha) x,
$$

where $x \in \mathbb{R}^{n}$ is the state and $A(\alpha) \in \mathbb{R}^{n \times n}$ is assumed to be in the polytope

$$
\mathcal{A}:=\left\{A(\alpha)=\sum_{i=1}^{N} \alpha_{i} A_{i}, \quad \alpha \in \Omega\right\},
$$

where the vector of uncertain parameters $\alpha \in \mathbb{R}^{N}$ is in

$$
\Omega=\left\{\sum_{i=1}^{N} \alpha_{i}=1, \quad \alpha_{i} \geq 0, \quad i=1, \ldots, N\right\} .
$$

In other words, any uncertain matrix $A(\alpha) \in \mathcal{A}$ can be written as the convex combination of the finite number of vertices $A_{i}, i=1, \ldots, N$.

The uncertain system (1) is said to be robustly stable if all matrices $A(\alpha)$ in $\mathcal{A}$ have eigenvalues with negative real part. By extension, we say that $A(\alpha)$ is robustly stable in $\mathcal{A}$ when system (1) is robustly stable. Clearly, $A(\alpha)$ is robustly stable in $\mathcal{A}$ if and only if there exists a parameter dependent quadratic Lyapunov function $V(\alpha, x)=x^{T} X(\alpha) x$, where $X(\alpha)=X(\alpha)^{T} \in \mathbb{R}^{n \times n}$, such that $V(\alpha, x)>0$ and $\dot{V}(\alpha, x)<0$ in all trajectories of system (1) for all $\alpha \in \Omega$ and $x \neq 0$.

In this paper, robust stability of the uncertain system (1) is verified using the particular polynomial parameter-dependent quadratic Lyapunov function

$$
V_{\kappa}(\alpha, x):=x^{T} X_{\kappa}(\alpha) x,
$$

where $\kappa \geq 1$ is an integer, $X_{\kappa}(\alpha) \in \mathbb{R}^{n \times n}$ is such that

$$
X_{\kappa}(\alpha):=A_{\kappa}(\alpha)^{T} P_{\kappa}(\alpha) A_{\kappa}(\alpha)>0,
$$

the matrix $A_{\kappa}(\alpha) \in \mathbb{R}^{\kappa n \times n}$ is defined by

$$
A_{\kappa}(\alpha):=\left[\begin{array}{c}
A(\alpha)^{0} \\
\vdots \\
A(\alpha)^{\kappa-1}
\end{array}\right]=\left[\begin{array}{c}
\mathrm{I}_{n} \\
\vdots \\
A(\alpha)^{\kappa-1}
\end{array}\right],
$$

and the matrix $P_{\kappa}(\alpha)=P_{\kappa}(\alpha)^{T} \in \mathbb{R}^{\kappa n \times \kappa n}$, which is a polynomial in $\alpha$, is partitioned in the form

$$
P_{\kappa}(\alpha):=\left[\begin{array}{ccc}
P_{11}(\alpha) & \cdots & P_{1 \kappa}(\alpha) \\
\vdots & \ddots & \vdots \\
P_{1 \kappa}(\alpha)^{T} & \cdots & P_{\kappa \kappa}(\alpha)
\end{array}\right],
$$

where each block $P_{j k}(\alpha) \in \mathbb{R}^{n \times n}$, for all $j, k=1, \ldots, \kappa$.

The reasons for assuming such particular parametrization for the polynomial Lyapunov matrix $X_{\kappa}(\alpha)$ will become clear in the next sections. Note that we do not require that $P_{\kappa}(\alpha)$ be positive definite or semi-definite, except for $\kappa=1$, when $A_{1}(\alpha)=\mathrm{I}_{n}$ and $X_{1}(\alpha)=P_{1}(\alpha)=P_{11}(\alpha) \in \mathbb{R}^{n \times n}$.
Definition 1 We say that the uncertain system (1) is $\kappa$ robustly stable or, equivalently, that $A(\alpha)$ is $\kappa$-robustly stable in $\mathcal{A}$ when there exists a polynomial parameter dependent matrix $X_{\kappa}(\alpha)>0$ in the form given in (5) such that

$$
A(\alpha)^{T} X_{\kappa}(\alpha)+X_{\kappa}(\alpha) A(\alpha)<0 .
$$

The following results reveal some features of this new notion of robust stability.

Lemma 1 If there exists an integer $\bar{\kappa} \geq 1$ such that $A(\alpha)$ is $\bar{\kappa}$-robustly stable in $\mathcal{A}$ then $A(\alpha)$ is $\kappa$-robustly stable in $\mathcal{A}$ for any integer $\kappa \geq \bar{\kappa}$.

Proof: If $A(\alpha)$ is $\bar{\kappa}$-robustly stable in $\mathcal{A}$ then (8) has some feasible solution $X_{\bar{\kappa}}(\alpha)>0$. Now for $\kappa=\bar{\kappa}+1$ we have

$$
\begin{aligned}
X_{\kappa}(\alpha)= & X_{\bar{\kappa}}(\alpha)+A(\alpha)^{\bar{\kappa}^{T}} P_{\kappa \kappa}(\alpha) A(\alpha)^{\bar{\kappa}} \\
& +A(\alpha)^{\bar{\kappa}^{T}}\left(\sum_{j=1}^{\bar{\kappa}} P_{j \kappa}(\alpha)^{T} A(\alpha)^{j-1}\right) \\
& +\left(\sum_{j=1}^{\bar{\kappa}} P_{j \kappa}(\alpha)^{T} A(\alpha)^{j-1}\right)^{T} A(\alpha)^{\bar{\kappa}} .
\end{aligned}
$$

Therefore, with

$$
P_{j \kappa}(\alpha)=0, \quad j=1, \ldots \kappa,
$$

we have that (8) has some feasible solution $X_{\kappa}(\alpha)=$ $X_{\bar{\kappa}}(\alpha)>0$, that is, $A(\alpha)$ is $\kappa$-robustly stable in $\mathcal{A}$. By induction, $A(\alpha)$ must be $\kappa$-robustly stable in $\mathcal{A}$ for any $\kappa>\bar{\kappa}$.

Lemma 2 If $A(\alpha)$ is not n-robustly stable in $\mathcal{A}$ then it is not $\kappa$-robustly stable in $\mathcal{A}$ for any integer $\kappa>n$.

Proof: The proof is by contradiction. Assume that $A(\alpha)$ is not $n$-robustly stable in $\mathcal{A}$ but it is $\kappa$-robustly stable in $\mathcal{A}$ for $\kappa=n+1$. Then there exist some $X_{\kappa}(\alpha)>0$ that is a feasible solution to (8), but no $X_{n}(\alpha)>0$ is a feasible solution to $(8)$. Note that

$$
\begin{aligned}
X_{\kappa}(\alpha)= & X_{n}(\alpha)+A(\alpha)^{{ }^{T}} P_{\kappa \kappa}(\alpha) A(\alpha)^{n} \\
& +A(\alpha)^{n T}\left(\sum_{j=1}^{n} P_{j \kappa}(\alpha)^{T} A(\alpha)^{j-1}\right) \\
& +\left(\sum_{j=1}^{n} P_{j \kappa}(\alpha)^{T} A(\alpha)^{j-1}\right)^{T} A(\alpha)^{n} .
\end{aligned}
$$

Let

$d(\lambda, \alpha)=\lambda^{n}+d_{1}(\alpha) \lambda^{n-1}+\cdots+d_{n}(\alpha)=\operatorname{det}\left(\lambda \mathrm{I}_{n}-A(\alpha)\right)$,

be the characteristic polynomial of $A(\alpha)$. Recall that $A(\alpha)$ is linear on $\alpha$, so that the coefficients $d_{i}(\alpha), i=1, \ldots, n$, are polynomials of degree $n$ in $\alpha$. By the Calley-Hamilton Theorem [18] we have that

$d(A(\alpha), \alpha)=A(\alpha)^{n}+d_{1}(\alpha) A(\alpha)^{n-1}+\cdots+d_{n}(\alpha) \mathrm{I}_{n}=0$, 
or, equivalently,

$$
A(\alpha)^{n}=-\sum_{j=1}^{n} d_{j}(\alpha) A(\alpha)^{n-j}=-J_{n}(\alpha)^{T} A_{n}(\alpha),
$$

where $J_{n}(\alpha) \in \mathbb{R}^{n^{2} \times n}$ is the polynomial matrix

$$
J_{n}(\alpha):=\left[\begin{array}{c}
d_{n}(\alpha) \mathrm{I}_{n} \\
\vdots \\
d_{1}(\alpha) \mathrm{I}_{n}
\end{array}\right] .
$$

Now define $\hat{P}_{n}(\alpha) \in \mathbb{R}^{n^{2} \times n}$ to be the polynomial matrix

$$
\hat{P}_{n}(\alpha):=\left[\begin{array}{c}
P_{1 \kappa}(\alpha) \\
\vdots \\
P_{n \kappa}(\alpha)
\end{array}\right]
$$

so that

$$
\hat{P}_{n}(\alpha)^{T} A_{n}(\alpha)=\sum_{j=1}^{n} P_{j \kappa}(\alpha)^{T} A(\alpha)^{j-1} .
$$

Using (5) to express $X_{n}(\alpha)$ and substituting the above expressions back into $X_{\kappa}(\alpha)$ we obtain

$$
X_{\kappa}(\alpha)=A_{n}(\alpha)^{T} \bar{P}_{n}(\alpha) A_{n}(\alpha),
$$

where

$$
\begin{aligned}
\bar{P}_{n}(\alpha):=P_{n}(\alpha)+ & J_{n}(\alpha) P_{\kappa \kappa} J_{n}(\alpha)^{T} \\
& +J_{n}(\alpha) \hat{P}_{n}(\alpha)^{T}+\hat{P}_{n}(\alpha) J_{n}(\alpha)^{T} .
\end{aligned}
$$

Note that $\bar{P}_{n}(\alpha)$ is a polynomial matrix which, along with the fact that $X_{n}(\alpha)=A_{n}(\alpha)^{T} \bar{P}_{n}(\alpha) A_{n}(\alpha)>0$ is a feasible solution to (8), contradicts the hypothesis that $A(\alpha)$ is not $n$-robustly stable in $\mathcal{A}$.

This proof can be generalized for any $\kappa>n+1$ by employing the Calley-Hamilton Theorem to express all powers of $A(\alpha)^{j}$ for all $j>n$ as linear combinations of $A(\alpha)^{j}, j=0, \ldots, n-1$.

\section{LIFTED CONDITIONS FOR $\kappa$-RoBUST STABILITY}

In this section we explore the particular structure of the polynomial matrix $X_{\kappa}(\alpha)$ to lift the inequality (8) into an inequality of larger dimensions. We will show later that these lifted inequalities can be converted into sufficient tests for $\kappa$ robust stability in the form of LMI. To perform the lift we use Finsler's Lemma, as in reference [19].

Lemma 3 (Finsler) Consider $\xi \in \mathbb{R}^{r}, Q \in \mathbb{R}^{r \times r}$ and $B \in$ $\mathbb{R}^{s \times r}$ such that $\operatorname{rank}(B)<r$. Then,

$$
\xi^{T} Q \xi<0, \quad \forall \xi \neq 0: \quad B \xi=0,
$$

if and only if one the following conditions holds:

a) $B_{\perp}^{T} Q B_{\perp}<0$, where $B_{\perp}$ is such that $B B_{\perp}=0$ and $B B^{T}+B_{\perp}^{T} B_{\perp}>0$;

b) $\exists V \in \mathbb{R}^{r \times s}: Q+V B+B^{T} V^{T}<0$.

Proof: See [19], [20].

The above lemma will be used in the sequel after identifying inequalities $X_{\kappa}(\alpha)>0$ and (8) with form a) of Lemma 3 .
The next theorem will constructed lifted versions of these inequalities in a space of larger dimensions as in form $b$ ).

Theorem 4 Let $\kappa \geq 1$. There exist $P_{\kappa}(\alpha) \in \mathbb{R}^{\kappa n \times \kappa n}$ and $Y(\alpha) \in \mathbb{R}^{\kappa n \times(\kappa-1) n}, Z(\alpha) \in \mathbb{R}^{(\kappa+1) n \times \kappa n}$ such that

$$
\begin{gathered}
P_{\kappa}(\alpha)>Y(\alpha) C_{\kappa-1}(\alpha)+C_{\kappa-1}(\alpha)^{T} Y(\alpha)^{T}, \\
Q_{\kappa}(\alpha)+Z(\alpha) C_{\kappa}(\alpha)+C_{\kappa}(\alpha)^{T} Z(\alpha)^{T}<0,
\end{gathered}
$$

where

$$
\begin{aligned}
Q_{\kappa}(\alpha):= & \left(L_{\kappa}^{T} \otimes \mathrm{I}_{n}\right) P_{\kappa}(\alpha)\left(R_{\kappa} \otimes \mathrm{I}_{n}\right) \\
& +\left(R_{\kappa}^{T} \otimes \mathrm{I}_{n}\right) P_{\kappa}(\alpha)\left(L_{\kappa} \otimes \mathrm{I}_{n}\right),
\end{aligned}
$$

and

$$
\begin{aligned}
C_{\kappa}(\alpha) & :=L_{\kappa} \otimes A(\alpha)-R_{\kappa} \otimes \mathrm{I}_{n}, \quad C_{0}(\alpha)=0_{n}, \\
L_{\kappa} & :=\left[\begin{array}{ll}
\mathrm{I}_{\kappa} & 0_{\kappa \times 1}
\end{array}\right], \\
R_{\kappa} & :=\left[\begin{array}{ll}
0_{\kappa \times 1} & \mathrm{I}_{\kappa}
\end{array}\right],
\end{aligned}
$$

for all $\alpha \in \Omega$ if and only if $A(\alpha)$ is $\kappa$-robustly stable in $\mathcal{A}$.

Proof: First prove that (10) is equivalent to $X_{\kappa}(\alpha)>0$. This is immediate in the case $\kappa=1$. When $\kappa>1$, use the fact that

$$
\left(C_{\kappa-1}(\alpha)\right)_{\perp}=A_{\kappa}(\alpha)
$$

and Lemma 3 to show that inequality (10) is equivalent to

$$
\begin{aligned}
& 0<\left(C_{\kappa-1}(\alpha)\right)_{\perp}^{T} P_{\kappa}(\alpha)\left(C_{\kappa-1}(\alpha)\right)_{\perp} \\
& =A_{\kappa}(\alpha)^{T} P_{\kappa}(\alpha) A_{\kappa}(\alpha)=X_{\kappa}(\alpha) .
\end{aligned}
$$

Now use Lemma 3 one more time to prove that inequality (11) is equivalent to

$$
\left(C_{\kappa}(\alpha)\right)_{\perp}^{T} Q_{\kappa}(\alpha)\left(C_{\kappa}(\alpha)\right)_{\perp}<0 .
$$

Then note that

$$
\begin{gathered}
0>\left(C_{\kappa}(\alpha)\right)_{\perp}^{T} Q_{\kappa}(\alpha)\left(C_{\kappa}(\alpha)\right)_{\perp} \\
=A_{\kappa+1}(\alpha)^{T}\left[\left(L_{\kappa}^{T} \otimes \mathrm{I}_{n}\right) P_{\kappa}(\alpha)\left(R_{\kappa} \otimes \mathrm{I}_{n}\right)\right. \\
\left.+\left(R_{\kappa}^{T} \otimes \mathrm{I}_{n}\right) P_{\kappa}(\alpha)\left(L_{\kappa} \otimes \mathrm{I}_{n}\right)\right] A_{\kappa+1}(\alpha) \\
=A_{\kappa}(\alpha)^{T} P_{\kappa}(\alpha) A_{\kappa}(\alpha) A(\alpha) \\
\quad+A(\alpha)^{T} A_{\kappa}(\alpha)^{T} P_{\kappa}(\alpha) A_{\kappa}(\alpha) \\
\quad=A(\alpha)^{T} X_{\kappa}(\alpha)+X_{\kappa}(\alpha) A(\alpha),
\end{gathered}
$$

to conclude the proof.

\section{A. LMI Sufficient Conditions for $\kappa$-Robust Stability}

In this section we provide computable stability tests for $\kappa$-robust stability. With that in mind, we assume that each entry of $P_{\kappa}(\alpha)$ is linear on $\alpha \in \Omega$. More specifically, that

$$
P_{\kappa}(\alpha)=\sum_{i=1}^{N} \alpha_{i}\left(P_{\kappa}\right)_{i},
$$

where $\left(P_{\kappa}\right)_{i}=\left(P_{\kappa}\right)_{i}^{T} \in \mathbb{R}^{\kappa n \times \kappa n}$ is partitioned in the form

$$
\left(P_{\kappa}\right)_{i}:=\left[\begin{array}{ccc}
P_{11 i} & \cdots & P_{1 \kappa i} \\
\vdots & \ddots & \vdots \\
P_{1 \kappa i}^{T} & \cdots & P_{\kappa \kappa i}
\end{array}\right], \quad i=1, \ldots, N,
$$


where each block $P_{j k i} \in \mathbb{R}^{n \times n}$, for all $j, k=1, \ldots, \kappa$ and $i=1, \ldots, n$.

The above assumption implies that $X_{\kappa}(\alpha)$ is a polynomial matrix of degree $2 \kappa-1$. The following corollaries provide sufficient $\kappa$-robust stability tests in the form of LMIs.

Corollary 5 Let $\kappa \geq 1$. If there exist constant matrices $Y \in \mathbb{R}^{\kappa n \times(\kappa-1) n}, Z \in \mathbb{R}^{(\kappa+1) n \times \kappa n}$ and $\left(P_{\kappa}\right)_{i}=\left(P_{\kappa}\right)_{i}^{T} \in$ $\mathbb{R}^{\kappa n \times \kappa n}, i=1, \ldots, N$, such that the LMIs

$$
\begin{gathered}
\left(P_{\kappa}\right)_{i}>Y\left(C_{\kappa-1}\right)_{i}+\left(C_{\kappa-1}\right)_{i}^{T} Y^{T}, \\
\left(Q_{\kappa}\right)_{i}+Z\left(C_{\kappa}\right)_{i}+\left(C_{\kappa}\right)_{i}^{T} Z^{T}<0,
\end{gathered}
$$

defined for all $i=1, \ldots, N$, where

$$
\begin{aligned}
&\left(Q_{\kappa}\right)_{i}:=\left(L_{\kappa}^{T} \otimes \mathrm{I}_{n}\right)\left(P_{\kappa}\right)_{i}\left(R_{\kappa} \otimes \mathrm{I}_{n}\right) \\
& \quad+\left(R_{\kappa}^{T} \otimes \mathrm{I}_{n}\right)\left(P_{\kappa}\right)_{i}\left(L_{\kappa} \otimes \mathrm{I}_{n}\right), \\
&\left(C_{\kappa}\right)_{i}:=L_{\kappa} \otimes A_{i}-R_{\kappa} \otimes \mathrm{I}_{n}, \\
&\left(C_{0}\right)_{i}=0_{n},
\end{aligned}
$$

have a feasible solution then $A(\alpha)$ is $\kappa$-robustly stable in $\mathcal{A}$.

Proof: This proof is a consequence of the linearity of $A(\alpha)$ and of the inequalities of Theorem 4 on $\alpha$ when $Y(\alpha)=Y$ and $Z(\alpha)=Z$. Indeed, multiply each inequality of the form (18) and (19) by the corresponding $\alpha_{i} \geq 0$, $i=1, \ldots, N$, and sum the resulting terms to obtain

$$
\begin{gathered}
P_{\kappa}(\alpha)>Y C_{\kappa-1}(\alpha)+C_{\kappa-1}(\alpha)^{T} Y^{T}, \\
Q_{\kappa}(\alpha)+Z C_{\kappa}(\alpha)+C_{\kappa}(\alpha)^{T} Z^{T}<0,
\end{gathered}
$$

which, from Theorem 4 , guarantees that $A(\alpha)$ is $\kappa$-robustly stable in $\mathcal{A}$.

Note that when $\kappa=1, X_{\kappa}(\alpha)=P_{11}(\alpha)$ is linear on $\alpha$, and the result of Corollary 5 coincides with that of [5].

Clearly, the above result comes from imposing the particular constant multipliers $Y(\alpha)=Y, Z(\alpha)=Z$ in Theorem 4. If we now impose that matrices $Y(\alpha)$ and $Z(\alpha)$ are linear in the uncertain parameter $\alpha$, that is

$$
Y(\alpha)=\sum_{i=1}^{N} \alpha_{i} Y_{i}, \quad Z(\alpha)=\sum_{i=1}^{N} \alpha_{i} Z_{i},
$$

the methodology of [6] can provide another sufficient $\kappa$ robust stability LMI condition, given in the next corollary.

Corollary 6 Let $\kappa \geq 1$. If there exist constant matrices $Y_{i} \in \mathbb{R}^{\kappa n \times(\kappa-1) n}, Z_{i} \in \mathbb{R}^{(\kappa+1) n \times \kappa n}$ and $\left(P_{\kappa}\right)_{i}=\left(P_{\kappa}\right)_{i}^{T} \in$ $\mathbb{R}^{\kappa n \times \kappa n}, i=1, \ldots, N$, such that the LMIs

$$
\begin{gathered}
\left(P_{\kappa}\right)_{i}>Y_{i}\left(C_{\kappa-1}\right)_{i}+\left(C_{\kappa-1}\right)_{i}^{T} Y_{i}^{T}, \\
\left(Q_{\kappa}\right)_{i}+Z_{i}\left(C_{\kappa}\right)_{i}+\left(C_{\kappa}\right)_{i}^{T} Z_{i}^{T}<0
\end{gathered}
$$

defined for all $i=1, \ldots, N$, and the LMIs

$$
\begin{aligned}
\left(P_{\kappa}\right)_{i} & +\left(P_{\kappa}\right)_{j} \\
> & Y_{i}\left(C_{\kappa-1}\right)_{j}+\left(C_{\kappa-1}\right)_{j}^{T} Y_{i}^{T} \\
& \quad+Y_{j}\left(C_{\kappa-1}\right)_{i}+\left(C_{\kappa-1}\right)_{i}^{T} Y_{j}^{T} \\
\left(Q_{\kappa}\right)_{i} & +\left(Q_{\kappa}\right)_{j} \\
& +Z_{i}\left(C_{\kappa}\right)_{j}+\left(C_{\kappa}\right)_{j}^{T} Z_{i}^{T} \\
& +Z_{j}\left(C_{\kappa}\right)_{i}+\left(C_{\kappa}\right)_{i}^{T} Z_{j}^{T}<0
\end{aligned}
$$

defined for all $i=1, \ldots, N-1$, and $j=i+1, \ldots, N$, where $\left(Q_{\kappa}\right)_{i},\left(C_{\kappa}\right)_{i}$ and $\left(C_{0}\right)_{i}$ have been defined in (20), (21) and (22), respectively, have a feasible solution then $A(\alpha)$ is $\kappa$-robustly stable in $\mathcal{A}$.

Proof: Use the linearity of $A(\alpha), P_{\kappa}(\alpha), Q_{\kappa}(\alpha), Y(\alpha)$ and $Z(\alpha)$, to write

$$
\begin{aligned}
& P_{\kappa}(\alpha)-Y(\alpha) C_{\kappa-1}(\alpha)-C_{\kappa-1}(\alpha)^{T} Y(\alpha)^{T} \\
& =\sum_{i=1}^{N} \alpha_{i}^{2}\left[\left(P_{\kappa}\right)_{i}-Y_{i}\left(C_{\kappa-1}\right)_{i}-\left(C_{\kappa-1}\right)_{i}^{T} Y_{i}^{T}\right] \\
& +\sum_{i=1}^{N-1} \sum_{j=i+1}^{N} \alpha_{i} \alpha_{j}\left[\left(P_{\kappa}\right)_{i}+\left(P_{\kappa}\right)_{j}-Y_{i}\left(C_{\kappa-1}\right)_{j}\right. \\
& \left.\quad-\left(C_{\kappa-1}\right)_{j}^{T} Y_{i}^{T}-Y_{j}\left(C_{\kappa-1}\right)_{i}-\left(C_{\kappa-1}\right)_{i}^{T} Y_{j}^{T}\right],
\end{aligned}
$$

and

$$
\begin{aligned}
Q_{\kappa}(\alpha) & +Z(\alpha) C_{\kappa}(\alpha)+C_{\kappa}(\alpha)^{T} Z(\alpha)^{T} \\
& =\sum_{i=1}^{N} \alpha_{i}^{2}\left[\left(Q_{\kappa}\right)_{i}+Z_{i}\left(C_{\kappa}\right)_{i}+\left(C_{\kappa}\right)_{i}^{T} Z_{i}^{T}\right] \\
+ & \sum_{i=1}^{N-1} \sum_{j=i+1}^{N} \alpha_{i} \alpha_{j}\left[\left(Q_{\kappa}\right)_{i}+\left(Q_{\kappa}\right)_{j}+Z_{i}\left(C_{\kappa}\right)_{j}\right. \\
& \left.+\left(C_{\kappa}\right)_{j}^{T} Z_{i}^{T}+Z_{j}\left(C_{\kappa}\right)_{i}+\left(C_{\kappa}\right)_{i}^{T} Z_{j}^{T}\right] .
\end{aligned}
$$

Inequalities (24-25) and (26-27) ensure that the conditions of Theorem 4 hold with $P_{\kappa}(\alpha), Y(\alpha)$ and $Z(\alpha)$ given respectively by (16) and (23).

The condition in Corollary 6 clearly encompasses the one proposed in Corollary 5, since when $Y_{i}=Y_{j}, Z_{i}=Z_{j}$, $i, j=1, \ldots, N$, inequalities (26-27) become trivial sums of the inequalities (24-25). Note also that when $\kappa=1$, the result of Corollary 6 is similar to the one presented in [8] for continuous-time robust stability.

\section{Illustrative EXAMPLES}

All numerical examples and experiments have been performed on a Pentium IV 2.6 GHz, 512 MB RAM, using SeDuMi [4], called from Matlab.

\section{A. Example I}

We borrowed an example from [11, Example 3], where $n=4$ and $N=3$. The maximum possible domain of stability, determined using brute force in [11], corresponds to the value $\hat{\rho}=2.2237$ (see [11] for details). Here we compare our results with those of [10], [11], [16].

For this example, the methods of [10], [11], [16] and Corollary 6 can successfully verify robust stability for the maximum domain of stability $\hat{\rho}$ under the following settings:

1) Set $k=2$ in [10, Corollary 4.4], to analyze robust stability of $N$ affine systems with $N-1$ uncertain parameters in $[-1,1]$.

2) Set $m=2$ in [11], to use a polynomial parameter dependent Lyapunov function of degree 2 .

3) Set $k=6$ in [16], to minimize a polynomial obtained from the Hermite matrix obtained at the 6 th relaxation 
iteration. To solve this problem we have used the software GloptiPoly (which calls SeDuMi [4] as a semidefinite program solver), with the additional constraint $\sum_{i} \alpha_{i}=1$.

4) Set $\kappa=2$ in Corollary 6.

Table I compares the computational effort required to solve the LMIs from Corollary 6 and to run the methods of [10], [11], [16]. Since the time required to solve a semi-definite program grows with the cube of the number of variables, the key to numerical efficiency seems to be able to keep the number of extra variables low. For instance, although the LMIs from Corollary 6 are twice as large as the ones from [11], the number of variables used by Corollary 6 is approximately half of the number of variables used by the method of [11]. Consequently, the LMIs from Corollary 6 can be solved five times faster than the ones from [11]. Also note that the methods of [11] and [16] require a significant amount of preliminary computation to build the LMIs. This extra time has not been included in the data shown in Table I.

TABLE I

\begin{tabular}{cccc}
\multicolumn{4}{c}{ EXAMPLE 3 FROM [11] $(n=4, N=3)$} \\
Number of \\
Method & Variables & Rows & Time (s) \\
\hline [10, Corollary 4.4$](k=2)$ & 572 & 52 & 8.66 \\
{$[11](m=2)$} & 750 & 64 & 10.97 \\
{$[16](k=6)$} & 454 & 476 & 29.27 \\
Corollary $6(\kappa=2)$ & 492 & 120 & 2.73 \\
\hline
\end{tabular}

\section{B. Numerical Experiments}

In this section we describe some numerical experiments we have performed to evaluate and compare the results of Corollaries 5 and 6.

For each $n=\{2,3,4\}$, and $N=\{2,3,4\}$ we have randomly generated a thousand stable polytopes, as described in [8]. We have then attempted to find feasible solutions to the LMIs given in Corollaries 5 and 6 for $\kappa=\{1,2,3\}$. The number of systems for which these tests have returned true is shown in Tables II and III. Some interesting facts emerge from the data:

1. Increasing $\kappa$ from 1 to 2 in Corollary 5 provides good improvement, while the improvement from increasing $\kappa$ from 2 to 3 seem to be marginal.

2. Corollaries 5 and 6 tested positive for all polytopes with $N=2$ and $\kappa \geq 2$. This might suggest that these conditions could be indeed necessary for $N=2$. This possibility is currently under investigation.

3. Corollary 6 tested positive for all polytopes with $\kappa \geq 2$.

We are also currently studying particular choices of the multipliers $Y$ and $Z$ in Corollaries 5 and 6 that might help to improve numerical efficiency while preserving the desirable low conservativeness of these methods as observed in Tables II and III.

\section{CONCLUSION}

We have proposed a new characterization of robust stability for continuous-time, linear time-invariant uncertain
TABLE II

NUMERICAL EXPERIMENT (COROLLARY 5)

\begin{tabular}{ccccc}
$n$ & $N$ & $\kappa=1$ & $\kappa=2$ & $\kappa=3$ \\
\hline \multirow{3}{*}{2} & 2 & 1000 & 1000 & 1000 \\
& 3 & 941 & 979 & 979 \\
& 4 & 798 & 814 & 817 \\
\hline \multirow{3}{*}{3} & 2 & 977 & 1000 & 1000 \\
& 3 & 717 & 790 & 790 \\
& 4 & 515 & 570 & 571 \\
\hline \multirow{3}{*}{4} & 2 & 978 & 1000 & 1000 \\
& 3 & 628 & 721 & 721 \\
& 4 & 397 & 452 & 453 \\
\hline
\end{tabular}

TABLE III

NUMERICAL EXPERIMENT (COROLLARY 6)

\begin{tabular}{ccccc}
$n$ & $N$ & $\kappa=1$ & $\kappa=2$ & $\kappa=3$ \\
\hline \multirow{3}{*}{2} & 2 & 1000 & 1000 & 1000 \\
& 3 & 1000 & 1000 & 1000 \\
& 4 & 1000 & 1000 & 1000 \\
\hline \multirow{3}{*}{3} & 2 & 977 & 1000 & 1000 \\
& 3 & 962 & 1000 & 1000 \\
& 4 & 965 & 1000 & 1000 \\
\hline \multirow{2}{*}{4} & 2 & 978 & 1000 & 1000 \\
& 3 & 952 & 1000 & 1000 \\
& 4 & 951 & 1000 & 1000 \\
\hline
\end{tabular}

systems that uses parameter-dependent quadratic Lyapunov functions which are polynomial on the uncertain parameter. The polynomial matrix that characterizes the quadratic Lyapunov matrix has been chosen to fit a particular form that depends on the dynamic matrix and on a symmetric matrix to be determined. Robust stability obtained with this particular Lyapunov function can be lifted into a larger dimensional space using Finsler's Lemma. In this larger space, we derived sufficient LMI conditions of increasing precision and complexity proportional to the degree of the polynomial matrix used in the quadratic Lyapunov function. The proposed LMI conditions performed results well when compared to other methods available in the literature. This is illustrated with numerical examples and comparative experiments.

\section{REFERENCES}

[1] B. R. Barmish, "Necessary and sufficient conditions for quadratic stabilizability of an uncertain system," Journal of Optimization Theory and Applications, vol. 46, no. 4, pp. 399-408, August 1985.

[2] S. Boyd, L. El Ghaoui, E. Feron, and V. Balakrishnan, Linear Matrix Inequalities in System and Control Theory. Philadelphia, PA: SIAM Studies in Applied Mathematics, 1994.

[3] P. Gahinet, A. Nemirovskii, A. J. Laub, and M. Chilali, LMI Control Toolbox User's Guide. The Math Works Inc., Natick, MA, 1995.

[4] J. F. Sturm, "Using SeDuMi 1.02, a MATLAB toolbox for optimization over symmetric cones," Optimization Methods and Software, vol. 11-12, pp. 625-653, 1999, URL: http://fewcal.kub.nl/sturm/software/sedumi.html.

[5] J. C. Geromel, M. C. de Oliveira, and L. Hsu, "LMI characterization of structural and robust stability," Lin. Alg. Appl., vol. 285, no. 1-3, pp. 69-80, December 1998.

[6] D. C. W. Ramos and P. L. D. Peres, "An LMI condition for the robust stability of uncertain continuous-time linear systems," IEEE Trans. Automat. Contr., vol. 47, no. 4, pp. 675-678, April 2002.

[7] D. Peaucelle, D. Arzelier, O. Bachelier, and J. Bernussou, "A new robust $\mathcal{D}$-stability condition for real convex polytopic uncertainty," Syst. Contr. Lett., vol. 40, no. 1, pp. 21-30, May 2000.

[8] V. J. S. Leite and P. L. D. Peres, "An improved LMI condition for robust $\mathcal{D}$-stability of uncertain polytopic systems," IEEE Trans. Automat. Contr., vol. 48, no. 3, pp. 500-504, March 2003. 
[9] Y. Ebihara and T. Hagiwara, "New dilated LMI characterizations for continuous-time multiobjective controller synthesis." Automatica, vol. 40, no. 11, pp. 2003-2009, November, 2004.

[10] P.-A. Bliman, "A convex approach to robust stability for linear systems with uncertain scalar parameters," SIAM J. Control Optim., vol. 42, no. 6, pp. 2016-2042, 2004.

[11] G. Chesi, A. Garulli, A. Tesi, and A. Vicino, "Robust stability of polytopic systems via polynomially parameter-dependent Lyapunov functions," in Proc. 42nd IEEE Conf. Decision Contr., Maui, HI, USA, December 2003, pp. 4670-4675.

[12] Y. Ebihara, K. Maeda, and T. Hagiwara, "Robust D-stability analysis of uncertain polynomial matrices via polynomial-type multipliers", in Proceedings of the 16th IFAC World Congress, Prague, CZ, July 2005.

[13] J. B. Lasserre, "Global optimization with polynomials and the problem of moments," SIAM J. Control Optim., vol. 11, no. 3, pp. 796-817, February 2001.

[14] P. A. Parrilo, "Semidefinite programming relaxations for semialgebraic problems." Mathematical Programming Ser. B, vol. 96, no. 2, pp. 293$320,2003$.
[15] G. Chesi, "Robust analysis of linear systems affected by time-invariant parametric uncertainty," in Proc. 42nd IEEE Conf. Decision Contr. Maui, HI, USA, December 2003, pp. 5019-5024.

[16] D. Henrion, D. Arzelier, D. Peaucelle, and J. B. Lasserre, "On parameter-dependent Lyapunov functions for robust stability of linear systems," in Proc. 43rd IEEE Conf. Decision Contr., Paradise Island, Bahamas, December 2004, pp. 887-892.

[17] C. W. Scherer and C. W. J. Hol, "Asymptotically exact relaxations for robust LMI problems based on matrix-valued sum-of-squares." In 16th Int. Symp. Math. Theory of Networks and Syst., Leuven, Belgium, July 2004.

[18] R. A. Horn and C. R. Johnson, Matrix Analysis. Cambridge University Press, 1985.

[19] M. C. de Oliveira and R. E. Skelton, "Stability tests for constrained linear systems," in Perspectives in Robust Control, ser. Lecture Notes in Control and Information Science, S. O. Reza Moheimani, Ed. New York: Springer-Verlag, 2001, vol. 268, pp. 241-257.

[20] R. E. Skelton, T. Iwasaki, and K. Grigoriadis, A Unified Algebraic Approach to Linear Control Design. Bristol, PA: Taylor \& Francis, 1998. 